\title{
Perfil dos casos de câncer bucal tratados em centro de alta complexidade em
}

\section{oncologia}

\author{
Profile of oral cancer cases treated in a high-complexity oncology center \\ Perfil de los casos de cáncer bucal atendidos en un centro de oncología de alta complejidad
}

Recebido: 27/08/2021 | Revisado: 01/09/2021 | Aceito: 08/09/2021 | Publicado: 09/09/2021

\author{
Sandra Lucia Vieira Ulinski \\ ORCID: https://orcid.org/0000-0002-6948-9110 \\ Universidade do Norte do Paraná, Brasil \\ E-mail: s.ulinski49@gmail.com \\ Jaqueline Akemi Yamashita \\ ORCID: https://orcid.org/0000-0003-0140-2805 \\ Universidade do Norte do Paraná, Brasil \\ E-mail: jaqueline-akemi16@hotmail.com \\ Jéssica Alves Gonçalves Siqueira \\ ORCID: https://orcid.org/0000-0002-7172-1580 \\ Universidade do Norte do Paraná, Brasil \\ E-mail: jessicaags192@hotmail.com \\ Alisson Gabriel Ildefonso Bistaffa \\ ORCID: https://orcid.org/0000-0003-2070-1472 \\ Universidade do Norte do Paraná, Brasil \\ E-mail: alissongabrielibistaffa@gmail.com \\ Antônio Plácido Peixoto do Amarante Neto \\ ORCID: https://orcid.org/0000-0002-8696-1042 \\ Instituto do Câncer de Londrina, Brasil \\ E-mail: amarante.neto@hotmail.com \\ Luiz Wanderley Romaniezen \\ ORCID: https://orcid.org/0000-0001-6745-8452 \\ Instituto do Câncer de Londrina, Brasil \\ E-mail: luizwr@sercomtel.com.br \\ Marcelo Lupion Poleti \\ ORCID: https://orcid.org/0000-0003-1904-5762 \\ Instituto Federal do Paraná, Brasil \\ E-mail: marcelo_poleti@yahoo.com.br \\ Thais Maria Freire Fernandes \\ ORCID: https://orcid.org/0000-0002-4368-8568 \\ Universidade do Norte do Paraná, Brasil \\ E-mail: thais.poleti@kroton.com.br
}

\begin{abstract}
Resumo
Objetivo: Descrever o perfil epidemiológico dos pacientes com câncer bucal tratados em um hospital de referência regional para tratamento de câncer no Paraná. Métodos: Este estudo retrospectivo, com delineamento transversal, envolveu uma população de 274 pessoas com câncer bucal, tratados no período de janeiro de 2007 a janeiro de 2014. Os dados foram coletados a partir dos prontuários hospitalares e complementados com as fichas de admissão hospitalar. Resultados: Identificou-se que 75,2\% eram indivíduos do gênero masculino, e $81,5 \%$ de cor/raça branca. A média de idade foi 59 anos, variando de 19 a 88 anos, com maior acometimento para a faixa etária com mais 60 anos com $48,2 \%$ dos casos. As pessoas mais acometidas pela doença exerciam atividades relacionadas à agricultura $(21,9 \%)$ e atividades relacionadas à produção de bens e serviços industriais (20,0\%). O tipo histológico mais prevalente foi o carcinoma espinocelular em 90,2\% casos, seguido pelo carcinoma basocelular (3,3\%) e adenocarcinoma (2,9\%). Conclusões: Os indivíduos mais acometidos foram homens, de raça branca, com idade média de 59 anos, com profissão ligada a agricultura, com lesões localizadas principalmente em lábio.
\end{abstract}

Palavras-chave: Carcinoma de células escamosas; Epidemiologia; Fatores de risco.

\begin{abstract}
Objective: To describe the epidemiological profile of oral cancer patients treated at a regional referral hospital for cancer treatment in Paraná. Methods: This retrospective, cross-sectional study involved a population of 274 people with oral cancer, treated from January 2007 to January 2014. Data were collected from hospital records and supplemented with admission forms from hospital. Results: It was identified that $75.2 \%$ were male, and $81.5 \%$ were white. The average age was 59 years old, ranging from 19 to 88 years old, with greater involvement for the age group over 60 years old
\end{abstract}


with $48.2 \%$ of cases. The people most affected by the disease performed activities related to agriculture (21.9\%) and activities related to production of industrial goods and services (20.0\%). The most prevalent histological type was squamous cell carcinoma in $90.2 \%$ of cases, followed by basal cell carcinoma (3.3\%) and adenocarcinoma $(2.9 \%)$. Conclusions: The most affected individuals were men, white, with an average age of 59 years, with a profession related to agriculture, with lesions located mainly on the lip.

Keywords: Carcinoma, squamous cell; Epidemiology; Risk factors.

\section{Resumen}

Objetivo: Describir el perfil epidemiológico de pacientes con cáncer bucal atendidos en un hospital regional de referencia para tratamiento oncológico en Paraná. Métodos: Este estudio retrospectivo, con un diseño transversal, involucró a una población de 274 personas con cáncer oral, atendidas desde enero de 2007 a enero de 2014. Los datos fueron recolectados de registros hospitalarios y complementados con formularios de admisión hospitalaria. Resultados: Se identificó que el 75,2\% eran varones y el 81,5\% eran blancos / de color. La edad promedio fue de 59 años, con un rango de 19 a 88 años, con mayor afectación para el grupo de edad mayor de 60 años con un 48,2\% de los casos. Las personas más afectadas por la enfermedad realizaban actividades relacionadas con la agricultura $(21,9 \%)$ y actividades relacionadas con la producción de bienes y servicios industriales $(20,0 \%)$. El tipo histológico más prevalente fue el carcinoma epidermoide en el 90,2\% de los casos, seguido del carcinoma basocelular $(3,3 \%)$ y el adenocarcinoma $(2,9 \%)$. Conclusiones: Los individuos más afectados fueron hombres, de raza blanca, con una edad promedio de 59 años, con una profesión relacionada con la agricultura, con lesiones localizadas principalmente en el labio.

Palabras clave: Carcinoma de células escamosas; Epidemiología; Factores de riesgo.

\section{Introdução}

O câncer bucal caracteriza-se como um relevante e crescente problema de saúde pública. Sua incidência vem aumentando e representa $3 \%$ dos casos considerando todos os tipos de câncer registrados anualmente no mundo. Isto se deve ao envelhecimento, ao crescimento populacional, e à mudança na distribuição e na prevalência dos fatores de risco de câncer, principalmente aos associados ao desenvolvimento socioeconômico (Sarode et al, 2020; Soares et al, 2019).

A incidência do câncer bucal no Brasil é considerada uma das mais elevadas do mundo. O Brasil apresentou 4.923 óbitos por câncer da cavidade oral em homens e 1.372 óbitos em mulheres, em 2017. Tais valores correspondem ao risco de 4,88/100 mil homens e 1,33/100 mil mulheres (Dhanuthai et al, 2018; Fonseca et al, 2014; INCA, 2014). O Instituto Nacional de Câncer José Alencar Gomes da Silva (INCA) estima que o número de casos novos de câncer bucal esperados para cada ano do triênio 2020-2022, será 11.180 e 4.010 casos em homens e mulheres, respectivamente.

No Paraná, as taxas estimadas de incidência de neoplasia maligna na cavidade bucal para 2020 é de 9,52 para cada 100.000 homens e 2,75 para cada 100.000 mulheres (INCA, 2020).

Várias estruturas anatômicas podem ser acometidas pelo câncer bucal, como: lábios, gengivas, bochechas, céu da boca, língua (principalmente as bordas) e a região embaixo da língua. Não existe um consenso nas literaturas nacional e internacional sobre quais localizações compõem a sua definição, sendo consideradas como câncer da cavidade oral aquelas localizadas nos lábios, cavidade oral, glândulas salivares e orofaringe (Cunha, Prass \& Hugo, 2020; Conway, Purkayastha, \& Chestnutt, 2018).

O câncer bucal tem etiologia multifatorial. A maior prevalência dos casos relaciona-se a fatores ambientais, sobretudo ao estilo de vida dos indivíduos. Dentre eles destacam-se: profissão, local de residência, nutrição e predisposição genética. Em relação aos hábitos pessoais, o uso de álcool e tabaco apresentam uma maior predisposição para neoplasias malignas em região de cabeça e pescoço. A exposição solar excessiva também pode ser um fator de risco considerável (Soares et al, 2019). Estudos tem identificado que a maioria dos casos ocorrem em indivíduos do sexo masculino, cor branca e faixa etária a partir de 50 anos de idade (American Cancer Society, 2019; Dantas et al, 2016; Fonseca et al, 2014; Soares et al, 2019).

O conhecimento das doenças bucais por meio de estudos epidemiológicos perfaz um importante papel na saúde pública e no que se refere à Estomatologia, revelando a precisão da prevalência, da incidência e da evolução de inúmeras doenças que acometem a boca, dentre elas o câncer bucal, bem como a distribuição percentual dentro de características próprias de determinadas populações regionais e mundiais. O diagnóstico precoce e tratamento imediato da doença são essenciais para a 
redução da morbidade e mortalidade (Torres et al, 2016).

Diante do perfil epidemiológico dos casos diagnosticados e tratados, é possível conhecer a realidade local e estabelecer uma proposta de plano de ação no combate ao câncer bucal aumentando o acesso ao serviço de prevenção e diagnóstico precoce no município e região. Sendo assim, o objetivo deste estudo é descrever o perfil epidemiológico do câncer bucal tratados nos últimos 7 anos em um hospital de referência regional para tratamento de câncer no Paraná.

\section{Metodologia}

Este estudo descritivo, retrospectivo, com delineamento transversal (Estrela, 2018), envolveu uma população de 274 pessoas com câncer bucal, tratados no Hospital do Câncer de Londrina de 2007 a janeiro de 2014. Esse hospital foi eleito por ser uma importante instituição de referência para o tratamento de câncer no interior do Paraná.

Os dados foram coletados a partir dos prontuários dos pacientes e complementados com as fichas de admissão hospitalar. Os prontuários foram selecionados independente do sexo, idade e tipo de tratamento.

Foram incluídos indivíduos cuja doença se enquadrava no Código Internacional de Doenças na classificação de neoplasias bucais (C00, C01, C02, C03, C04, C05, C06, C07, C08, C09 e C10).

Foram encontrados 298 prontuários. Destes, 274 foram selecionados. Os prontuários que não apresentavam os dados de localização e tipo histopatológico foram excluídos.

As variáveis do estudo foram: ano de admissão hospitalar, idade, sexo, raça, ocupação (Classificação Brasileira de Ocupações - Quadro 1), cidade de origem do paciente, localização do câncer, tipo histopatológico, estadiamento e tratamento antineoplásico.

Quadro1 - Classificação Brasileira de Ocupações.

\begin{tabular}{|l|l|}
\hline \multicolumn{2}{|l|}{ CBO - Grandes Grupos / Títulos } \\
\hline 0 & Forças Armadas, Policiais e Bombeiros Militares \\
\hline 1 & Membros superiores do poder público, dirigentes de organizações de interesse público e de empresas e gerentes \\
\hline 2 & Profissionais das ciências e das artes \\
\hline 3 & Técnicos de nível médio \\
\hline 4 & Trabalhadores de serviços administrativos \\
\hline 5 & Trabalhadores dos serviços, vendedores do comércio em lojas e mercados \\
\hline 6 & Trabalhadores agropecuários, florestais, da caça e pesca \\
\hline 7 & Trabalhadores da produção de bens e serviços industriais \\
\hline 8 & Trabalhadores da produção de bens e serviços industriais \\
\hline 9 & Trabalhadores de manutenção e reparação \\
\hline
\end{tabular}

Fonte: Brasil, 2021. Disponível em: http://www.mtecbo.gov.br/cbosite/pages/home.jsf.

A análise estatística foi utilizada para determinar a distribuição de frequência das variáveis, seguida pela análise bivariada utilizando tabelas de contingência (Yin, 2015).

O projeto foi aprovado pelo Comitê de Ética Em Pesquisa da Universidade Norte do Paraná, sob o nº704.341, em 26/06/2014.

\section{Resultados}

Os dados levantados de 274 pacientes com câncer bucal, tratados de2007 a 2014, estão apresentados na Tabela 1. 
Tabela 1 - Dados sociodemográficos e localização da lesão dos pacientes tratados no período de 2007 a 2014.

\begin{tabular}{|c|c|c|c|c|c|c|c|}
\hline & \multicolumn{2}{|c|}{ Feminino } & \multicolumn{2}{|c|}{ Masculino } & \multicolumn{2}{|l|}{ Total } & \multirow[b]{2}{*}{$\mathrm{p}$} \\
\hline FAIXA ETÁRIA & $\mathrm{n}$ & $\%$ & $\mathrm{n}$ & $\%$ & $\mathrm{n}$ & $\%$ & \\
\hline 10 a 19 anos & & & 1 & 0,5 & 1 & 0,4 & 0,080 \\
\hline 20 a 29 anos & 2 & 2,9 & 3 & 1,5 & 5 & 1,8 & \\
\hline 30 a 39 anos & 4 & 5,9 & 7 & 3,4 & 11 & 4,0 & \\
\hline 40 a 49 anos & 5 & 7,4 & 32 & 15,5 & 37 & 13,5 & \\
\hline 50 a 59 anos & 18 & 26,5 & 70 & 34,0 & 88 & 32,1 & \\
\hline 60 a 69 anos & 14 & 20,6 & 51 & 24,8 & 65 & 23,7 & \\
\hline Mais de 70 anos & 25 & 36,8 & 42 & 20,4 & 67 & 24,3 & \\
\hline \multicolumn{8}{|l|}{ COR/RAÇA } \\
\hline Amarela & 2 & 3,2 & & & 2 & 0,8 & 0,024 \\
\hline Branca & 53 & 85,5 & 150 & 80,2 & 203 & 81,5 & \\
\hline Negra & 3 & 4,8 & 7 & 3,7 & 10 & 4,0 & \\
\hline Parda & 4 & 6,5 & 30 & 16,0 & 34 & 13,65 & \\
\hline \multicolumn{8}{|l|}{$\mathrm{CBO}$} \\
\hline 1 & 0 & 0,0 & 8 & 3,9 & 8 & 2,9 & 0,00 \\
\hline 2 & 5 & 7,4 & 5 & 2,4 & 10 & 3,7 & \\
\hline 3 & 1 & 1,5 & 3 & 1,5 & 4 & 1,5 & \\
\hline 4 & 0 & 0,0 & 10 & 4,9 & 10 & 3,7 & \\
\hline 5 & 4 & 5,9 & 22 & 10,7 & 26 & 9,5 & \\
\hline 6 & 4 & 5,9 & 56 & 27,2 & 60 & 21,9 & \\
\hline 7 & 5 & 7,4 & 50 & 24,3 & 55 & 20,1 & \\
\hline 8 & 0 & 0,0 & 11 & 5,3 & 11 & 4,0 & \\
\hline 9 & 8 & 11,8 & 25 & 12,1 & 33 & 12,0 & \\
\hline Não trabalha ${ }^{1}$ & 34 & 50,0 & 1 & 0,5 & 35 & 12,8 & \\
\hline \multicolumn{8}{|l|}{ Localização } \\
\hline Amigdala & 0 & - & 2 & 1,0 & 2 & 0,7 & 0,241 \\
\hline Área Retromolar & 2 & 2,9 & 3 & 1,5 & 5 & 1,8 & \\
\hline Assoalho de Boca & 6 & 8,8 & 34 & 16,5 & 40 & 14,6 & \\
\hline Cavidade Oral & 1 & 1,5 & 1 & 0,5 & 2 & 0,7 & \\
\hline Gengiva & 5 & 7,4 & 9 & 4,4 & 14 & 5,1 & \\
\hline Gl. Sublingual & 1 & 1,5 & & & 1 & 0,4 & \\
\hline Lábio & 17 & 25,0 & 53 & 25,7 & 70 & 25,6 & \\
\hline Língua & 12 & 17,7 & 51 & 24,8 & 63 & 23,0 & \\
\hline Mandíbula & 0 & - & 2 & 1,0 & 2 & 0,7 & \\
\hline Maxila & 2 & 2,9 & 0 & - & 2 & 0,7 & \\
\hline Mucosa Jugal & 1 & 1,5 & 5 & 2,4 & 6 & 2,2 & \\
\hline Orofaringe & 3 & 4,4 & 8 & 3,9 & 11 & 4,0 & \\
\hline Palato & 18 & 26,5 & 35 & 17,0 & 53 & 19,3 & \\
\hline Rebordo inferior & 0 & - & 1 & 0,5 & 1 & 0,4 & \\
\hline Seio Nasal & 0 & - & 1 & 0,5 & 1 & 0,4 & \\
\hline Submentoniano & 0 & - & 1 & 0,4 & 1 & 0,4 & \\
\hline
\end{tabular}

1 do lar ou estudante.

Fonte: Dados da pesquisa (2020).

Do total da amostra estudada, identificou-se que 75,2\% eram do gênero masculino, numa proporção homem/mulher 3:1. 
Indivíduos de cor/raça branca constituíram 81,5\% dos casos. A média de idade foi 59 anos, variando de 19 a 88 anos, com maior acometimento para a faixa etária com mais 60 anos com $48,2 \%$ dos casos.

Observou-se iniquidades em relação à função laboral das pessoas. As pessoas mais acometidas pela doença exerciam atividades relacionadas à agricultura $(21,9 \%)$ e atividades relacionadas à produção de bens e serviços industriais (20,0\%). Por outro lado, aquelas que ocupavam cargos de nível técnico e cargos de direção ou ligados ao setor público (2,9\%), 1,5\% e 2,9\% respectivamente, representando $4,4 \%$ dos casos.

As regiões topográficas mais atingidas foram o lábio (25,6\%), seguido da língua (23\%) e do palato (19,3\%).

$\mathrm{Na}$ Tabela 2, observa-se que o tipo histológico mais prevalente foi o carcinoma espinocelular em 90,2\% casos, seguido pelo carcinoma basocelular $(3,3 \%)$ e adenocarcinoma $(2,9 \%)$.

Tabela 2 - Prevalência de câncer segundo tipo histopatológico.

\begin{tabular}{lcc}
\hline TIPO & Frequência & Percentual \\
\hline Adenocarcinoma & 8 & $2,9 \%$ \\
Carcinoma Adenóide Cístico & 5 & $1,8 \%$ \\
Carcinoma Basocelular & 9 & $3,3 \%$ \\
Carcinoma Intraepitelial & 1 & $0,4 \%$ \\
Carcinoma espinocelular & 247 & $90,1 \%$ \\
Neoplasia Maligna & 4 & $1,5 \%$ \\
Total & 274 & $100,00 \%$ \\
\hline
\end{tabular}

Fonte: Dados da pesquisa (2020).

Em relação ao estadiamento (Tabela 3), foi evidenciado que 41,6\% dos casos foram diagnosticados com estadiamento II e somente 17,31\% dos casos com estadiamento I. Identificou-se que nos anos de 2011, 2012 e 2013 o diagnóstico dos casos foi muito tardio, pois predominou o estadiamento IV. Dentre os tratamentos recebidos, predominou a cirurgia $(34,7 \%)$, a radioterapia $(16,4 \%)$ e a cirurgia seguida de radioterapia $(8,8 \%)$.

Tabela 3 - Estadiamento clínico dos casos diagnosticados de câncer.

\begin{tabular}{lrr}
\hline ESTADIAMENTO & Frequência & Percentual \\
\hline I & 36 & $17,31 \%$ \\
II & 72 & $34,62 \%$ \\
III & 35 & $16,83 \%$ \\
IV & 30 & $14,42 \%$ \\
Óbito & 35 & $16,83 \%$ \\
Total & 208 & $100,00 \%$ \\
\hline
\end{tabular}

Fonte: Dados da pesquisa (2020).

As maiores taxas de ocorrência foram nos anos de 2007 e 2009, com 32,1\% e 16,8\% dos casos. Na Figura 1, identificase uma tendência de redução no número de casos admitidos no hospital. 
Figura 1 - Distribuição do número de casos, conforme o ano de admissão no hospital.

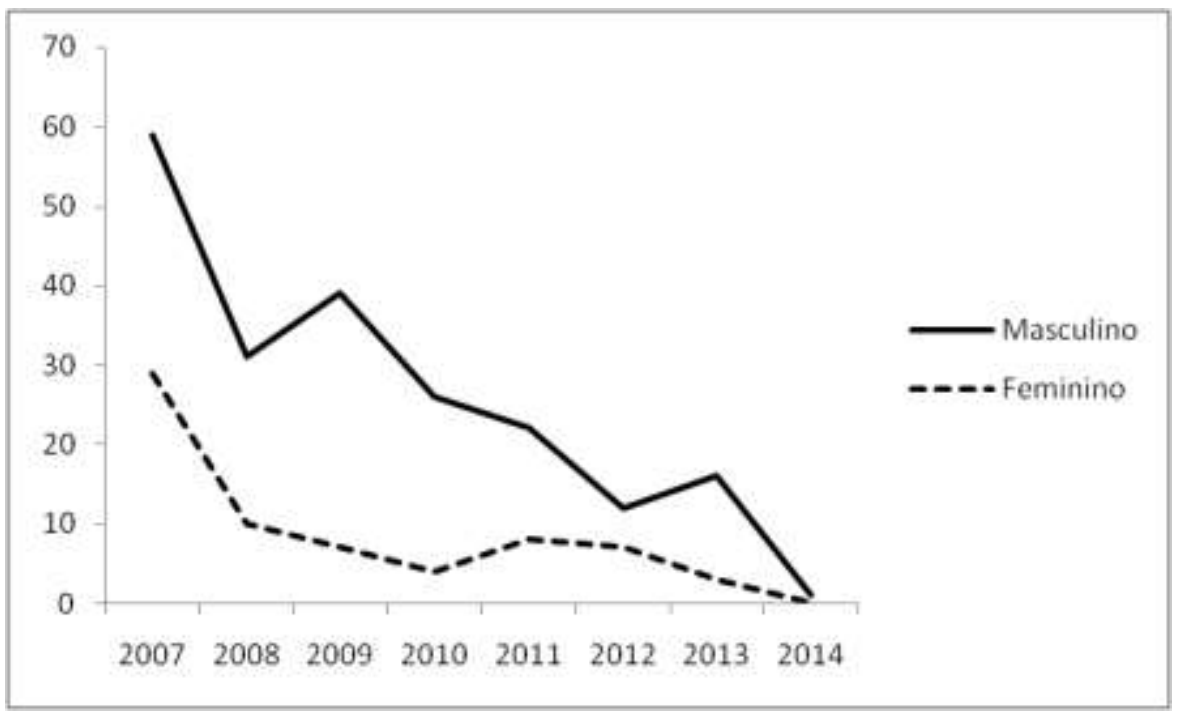

Fonte: Dados da pesquisa (2020).

\section{Discussão}

Este estudo identificou 274 casos de câncer bucal no período de 2007 a 2014 tratados em hospital de referência para câncer. O perfil caracterizado por homens brancos (Dias et al, 2017), trabalhando em profissões que não exigem qualificações especializadas, infere uma maior vulnerabilidade à doença (Soares et al, 2019). Achados indicam que pode ocorrer risco aumentado de câncer bucal em jovens devido ao tabagismo por longo período de tempo e ao consumo de álcool (Dantas et al, 2016)

Referente à idade que pacientes são diagnosticados com câncer bucal a literatura relata que o grupo de 55 a 64 anos é o mais atingido (Dias et al, 2017). Estudos mostram que o gênero mais atingido com o câncer bucal é o masculino e a localização mais prevalente na região da língua (Dantas et al, 2016).

O tipo histológico mais prevalente de câncer bucal encontrado no estudo foi o carcinoma espinocelular (Borba Ribeiro et al, 2019; Dias et al, 2017). A literatura aponta que, dentre os tumores malignos que se desenvolvem na cavidade bucal, esta é a neoplasia maligna de maior incidência, com um percentual de 90\% a 95\% dos casos (Dantas et al, 2016; Silva et al, 2019). O carcinoma espinocelular tem etiologia multifatorial, com fatores endógenos (predisposição genética) e fatores exógenos ambientais e comportamentais (tabagismo, etilismo, exposição excessiva ao sol, higiene bucal inadequada, desnutrição, imunodeficiências e infecções secundárias) (Leite et al, 2021; Silva et al, 2019).

Predominou, nesse estudo, as atividades ligadas a agricultura (Dias et al, 2017) e a (trabalhadores da produção de bens e serviços industriais). Entre as mulheres predominou a função doméstica. Acerca da função laboral, existem escassas pesquisas no Brasil, no entanto alguns estudos tem demonstrado relações significativas com o risco de desenvolver câncer bucal. Tem se observado que trabalhadores ao ar livre, como pescadores e agricultores, apresentam maior risco de desenvolver câncer bucal no lábio, associado à exposição à radiação ultravioleta. Além disso, o uso de agrotóxicos também se apresenta como um fator de risco ocupacional desses trabalhadores (Castro et al, 2019).

Ganesh et al. (2013), evidenciaram que a maior parcela da população de seu estudo sobre o perfil de câncer bucal como trabalhadores rurais ou trabalhadores urbanos não qualificados, com renda familiar muito baixa e altas taxas de analfabetismo em ambos os casos. Tal fato indica que existe um excesso de exposição de trabalhadores de classes econômicas mais baixas e populações das periferias das grandes cidades aos riscos presentes nos processos produtivos (Conway, Purkayastha, \& Chestnutt, 2018). 
O lábio foi a região anatômica mais acometida. Isto se deve provavelmente às características ocupacionais, visto que uma parcela significativa dos casos trabalhava em atividades ligadas a agricultura. (Castro et al, 2019).

Identificou-se que os casos de câncer bucal foram diminuindo no decorrer dos anos, sendo que em 2007 e 2013 foram 73 e 13 casos detectados, respectivamente. No entanto, o nível de estadiamento na ocasião do diagnóstico do paciente foi no sentido inverso, aumentando sua severidade (Torres et al, 2016; Santos; Batista e Cangussu, 2010). Esses resultados inferem que a atenção com o diagnóstico precoce de câncer bucal pode ter diminuído. Estudos demonstram que uma parcela significativa dos casos diagnosticados da doença é identificada em sua fase avançada, em indivíduos de pouca renda, com baixa escolaridade e com dificuldade de acesso aos serviços de saúde. Destaca-se que a detecção precoce do câncer é fator determinante do prognóstico do câncer bucal. Tal fato remete à importância do acesso aos serviços de saúde na atenção primária. Torna-se necessário um esforço maior no sentido aumentar o acesso aos serviços de saúde bucal para a população. Reforçar as ações de detecção precoce permite maiores chances de cura e um aumento da sobrevida dos pacientes, especialmente entre os idosos (Raimundo et al, 2019).

Outro fator a considerar é a dificuldade do diagnóstico precoce, pois as lesões iniciais geralmente são assintomáticas. Essas passam despercebidas pelo indivíduo e profissional, demonstrando a falta de conhecimento da patologia e demora na procura do atendimento por parte do paciente. Esses aspectos demonstram a importância de fortalecer as políticas públicas voltadas para a detecção e tratamento do câncer bucal (Santos; Batista e Cangussu, 2010).

A crescente incidência do câncer bucal, em indivíduos com mais de 50 anos, traz a necessidade de incluir um exame sistematizado para essa população, integrando a rotina de atendimento das equipes de saúde. Tal ação provavelmente reduziria o número de casos com maior severidade de estadiamento do câncer bucal (Miranda et al, 2019).

Em relação à detecção precoce do câncer bucal, o INCA atualizou as diretrizes. O Instituto deixou de recomendar o autoexame e o rastreamento populacional. Essas novas diretrizes foram estabelecidas após a avaliação de estudos e pesquisas recentes, que apontaram a ausência de evidências científicas que tais medidas diminuíram a incidência ou a taxa de mortalidade pela doença (Atty et al, 2012).

A grande parcela da população não diferencia lesões potencialmente malignas de áreas anatômicas normais. Sem identificar a diferença, o indivíduo pode negligenciar lesões potencialmente perigosas, o que pode resultar em um diagnóstico tardio da doença e desfavorecer a deteç̧ão precoce. A recomendação do INCA é a procura imediata de um dentista ou médico no caso de surgir lesão na boca que não cicatrize em até 15 dias (Atty, 2012).

\section{Considerações Finais}

Este estudo registrou 274 casos de câncer bucal no período de 2007 a 2014 tratados em hospital de referência para câncer, com predomínio de homens brancos, trabalhando em profissões que não exigem qualificações. Ainda, identificou-se que o câncer bucal vem sendo diagnosticado tardiamente, apesar das políticas públicas nacionais direcionadas para esta doença. Por ser de fácil visualização ao exame físico, deduz-se que há uma lacuna de grande relevância nos diagnósticos desses carcinomas. As equipes de saúde, principalmente o cirurgião-dentista, tem um papel relevante na identificação clínica das lesões orais e na orientação à população para que evite os fatores de risco para o câncer bucal. Torna-se necessário também, um protagonismo das entidades de classe na prevenção e detecção do câncer bucal.

Para um melhor entendimento, recomenda-se estudos futuros mais aprofundados em relação aos fatores relacionados ao trabalho com o câncer bucal na população. 


\section{Referências}

American Cancer Society. (2019). Cancer facts \& figures 2019. American Cancer Society. rgiris, A., Karamouzis, M. V., Raben, D., \& Ferris, R. L. (2008). Head and neck cancer. The Lancet, 371(9625), $1695-1709$.

Atty, A., Pontes, J. R. M., Torres-Pereira, C., Lemos Junior, C. A., Vidal, A. K. L., \& Kulcsar, M. A. (2012). Novas diretrizes para a detecção precoce do câncer de boca. Rede Câncer, 19(1), 28-30.

Borba Ribeiro, K. R., Lira Júnior, C., Marinho, S. A., de Carvalho, S. H. G., Agripino, G. G., \& Sarmento, D. J. D. S. (2019). Epidemiological profile of young patients with squamous cell carcinoma in northeast Brazil. Journal of investigative and clinical dentistry, $10(4)$, e12436.

Conway, D. I., Purkayastha, M., \& Chestnutt, I. G. (2018). The changing epidemiology of oral cancer: definitions, trends, and risk factors. British dental journal, 225(9), 867-873.

Cunha, A. R. D., Prass, T. S., \& Hugo, F. N. (2020). Mortalidade por câncer bucal e de orofaringe no Brasil, de 2000 a 2013 : tendências por estratos sociodemográficos. Ciência \& Saúde Coletiva, 25, 3075-3086.

Dantas, T. S., de Barros Silva, P. G., Sousa, E. F., Cunha, M. D. P., de Aguiar, A. S. W., Costa, F. W. G., \& Sousa, F. B. (2016). Influence of educational level, stage, and histological type on survival of oral cancer in a Brazilian population: a retrospective study of 10 years observation. Medicine, 95(3).

Dhanuthai, K., Rojanawatsirivej, S., Thosaporn, W., Kintarak, S., Subarnbhesaj, A., Darling, M., Kryshtalskyj, E., Chiang, C., Shin, H., Choi S., Lee, S., \& Shakib, P. A. (2018). Oral cancer: A multicenter study. Medicina oral, patologia oral y cirugia bucal, 23(1), e23. ,

Dias, A. K. S., Diniz, M. F., Grangeiro, M. T. V., de Araujo, T. L., \& de Sousa Feitosa, D. A. (2017). Epidemiological profile of diagnosed injuries in bucal cancer: study of a brazilian population. Age, 44, 45-54.

Estrela, C. (2018). Metodologia científica: ciência, ensino, pesquisa. Artes Médicas.

Ganesh, R., John, J., \& Saravanan, S. (2013). Socio demographic profile of oral cancer patients residing in Tamil Nadu-A hospital based study. Indian journal of cancer, 50(1), 9 .

INCA. (2020) Estatísticas do Câncer. http://www.inca.gov.br/estimativa/2012/tabelasestados.asp?UF=BR.

INCA. (2020) Instituto Nacional do Câncer. Estimativa 2020. https://www.inca.gov.br/estimativa/introducao.

Fonseca, E. P., da Fonseca, S. G. O., Milagres, C. S., \& Lopes, A. G. (2014). Mortalidade por câncer bucal e orofaringe no Brasil entre 2002 e 2011. Pensar Acadêmico, 11(2), 8-17.

Leite, R. B., Marinho, A. C. O., Costa, B. L., Laranjeira, M. B. V., Araújo, K. D., \& Cavalcanti, A. F. (2021). A influência da associação de tabaco e álcool no câncer bucal: revisão de literatura. Jornal Brasileiro de Patologia e Medicina Laboratorial, 57.

Miranda, F. A., De Araujo, L. O., Melo, M. R., Barbosa, R. C., Caldeira, A. P., \& Oliveira, F. P. S. L. (2019). Políticas públicas em saúde relacionadas ao diagnóstico precoce e rastreamento do câncer bucal no brasil. SANARE-Revista de Políticas Públicas, 18(2).

Raimundo, D. D., Costa, T. C. D., Cunha, M. L. D., Silva, L. D. M. S., Hanzelmann, R. D. S., Granadeiro, D. D. S., ... \& Passos, J. P. (2019). Manifestações clínicas e sociodemográficas em trabalhadores com câncer bucal. Rev Enferm Ufpe On Line, Recife, 13(5), $1412-1419$.

Santos, L. C. O. D., Batista, O. D. M., \& Cangussu, M. C. T. (2010). Caracterização do diagnóstico tardio do câncer de boca no estado de Alagoas. Brazilian Journal of Otorhinolaryngology, 76, 416-422.

Sarode, G., Maniyar, N., Sarode, S. C., Jafer, M., Patil, S., \& Awan, K. H. (2020). Epidemiologic aspects of oral cancer. Disease-a-Month, 66(12), 100988.

Silva, A. A. F., da Silva Barros, C. C., de Morais, E. F., Pinheiro, J. C., Barboza, C. A. G., \& de Arruda, M. D. L. S. (2019). Perfil clínico-epidemiológico do Carcinoma Epidermoide Oral em pacientes adultos jovens dos 20 aos 45 anos. Revista da Faculdade de Odontologia-UPF, 24(1), 89-95.

Soares, É. C., Neto, B. C. B., \& de Souza Santos, L. P. (2019). Estudo epidemiológico do câncer de boca no Brasil/Epidemiological study of oral cancer in Brazil. Arquivos Médicos dos Hospitais e da Faculdade de Ciências Médicas da Santa Casa de São Paulo, 64(3), 192-198.

Torres, S. V., Sbegue, A., \& Costa, S. C. B. (2016). A importância do diagnóstico precoce de câncer bucal em idosos. Rev Soc Bras Clin Med, 14(1), 57-62.

Yin, R.K. (2015). O estudo de caso. Bookman. 